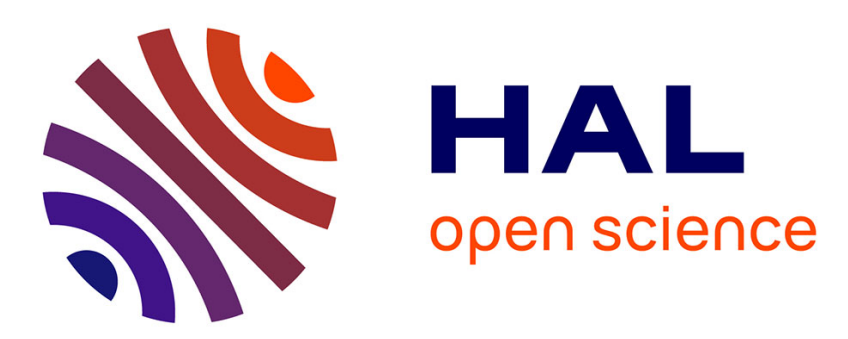

\title{
How to gain emotional rewards during human-robot interaction using music? Formulation and propositions
}

Thi-Haï-Ha Dang, Guillaume Hutzler, Philippe Hoppenot

\section{To cite this version:}

Thi-Haï-Ha Dang, Guillaume Hutzler, Philippe Hoppenot. How to gain emotional rewards during human-robot interaction using music? Formulation and propositions. 10th international conference on Artifical intelligence and soft computing: Part II (ICAISC 2010), Jun 2010, Zakopane, Poland. pp.247-255. hal-00604528

\section{HAL Id: hal-00604528 \\ https://hal.science/hal-00604528}

Submitted on 2 Dec 2013

HAL is a multi-disciplinary open access archive for the deposit and dissemination of scientific research documents, whether they are published or not. The documents may come from teaching and research institutions in France or abroad, or from public or private research centers.
L'archive ouverte pluridisciplinaire HAL, est destinée au dépôt et à la diffusion de documents scientifiques de niveau recherche, publiés ou non, émanant des établissements d'enseignement et de recherche français ou étrangers, des laboratoires publics ou privés. 


\title{
How to gain emotional rewards during human-robot interaction using music? Formulation and propositions
}

\author{
Thi-Hai-Ha Dang, Guillaume Hutzler, and Philippe Hoppenot \\ Laboratory IBISC, University of Evry Val d'Essonne, Boulevard Francois Mitterrand \\ 91025 Evry, France, \\ \{hadang, guillaume.hutzler, philippe.hoppenot\}@ibisc.univ-evry.fr
}

\begin{abstract}
In this paper, we present arguments for the need of emotion modelling and we define elements for a study in Human-Robot Interaction (HRI) using music. We also propose an adaptation of our generic model of emotions (GRACE) to give a precise idea of how to design emotional intelligence for a robot with music-related abilities.
\end{abstract}

\section{Introduction}

Thanks to the rapid development of artificial intelligence (including pattern recognition, speech processing, motion planning, machine learning, etc.), robots nowadays have gained various abilities to do repetitive, meticulous, dangerous tasks with high precision at great speed. Robots are thus greatly appreciated for replacing human in everyday/industrial/outer space tasks.

Besides, robotic applications are also present in assistive tasks, where comfortable experience is in the first rank of exigencies. This entails demands on soft skills for robots in order to deal with human mental states, to mimic human social interaction and to gain cooperation of human. Experimentations of robotic applications have also shown the emotional rewards to human in interacting with intelligent robots.

Moreover, various researches interested in music and emotions have claimed that the emotional reward of music remains an important reason for the universal appeal of music $[2,4]$. So, can music be involved in human-robot interaction to gain emotional rewards to human? This paper is an attempt to formulate such a case study.

In section two, we highlight the main characteristics of HRI to be taken into account in robotic application design. We present also in this section the findings of emotional rewards during human-robot interaction and recent works on emotion modelling in computer science. In section three, the first subsection lists important elements to be focused on in studying the role of emotional rewards in HRI using music. In the second subsection, we propose an adaptation of GRACE, our generic model of emotions, in order to take into account these elements. Finally, in section four, we give some perspectives for our study on emotional rewards in HRI using music. 


\section{The State of the Art}

Robotics, since the use of diverse robotic applications in human life, has been opening large perspectives of multi-disciplinary works, e.g. machinery design, control mechanism, beautiful appearance, intelligent reaction, good performance of task accomplishment and also efficient interface of interaction (i.e. humanrobot interaction - HRI). This HRI itself is also a field of study where several researches have been focused. The number of research projects on communication channels between human and robot (keyboard, mouse, voice, speech, gesture, etc.) increases with time. Many of them have gained successes in improving communication efficiency. Soon afterward, importance of the emotional aspect of communication began to draw attention. Researchers in HRI started to study/apply knowledge in emotion theories into their applications. In this section, we will present advances in HRI research and the need of emotion modelling in robotic applications.

\subsection{Human-Robot Interaction - Need of emotion modelling}

Natural and affective interaction - this could be considered as the principle goal of all works on human-machine interaction. Being a specific branch of research in human-machine interaction, research in HRI, specially HRI for robotic assistance, is focused on such a kind of interaction. Kiesler and Hinds, in the introduction of Special Issues in Human-Robot Interaction [10], mention some important points to be considered in HRI research, particularly in robotic assistance, which are:

- Anthropomorphic model: this relates to the need of using natural channels of communication to facilitate the interaction of humans with the robot. The more natural human-robot communication is, the better the robot gains cooperation from human. Several channels could be considered such as voice, speech, gesture, etc.

- Physically close to other robots, people, objects: usually, robotic assistance involves human participation in a close distance. This characteristic has to be seen as of major importance to study HRI. This importance appears in the necessity of robots having abilities to work in a dynamic and challenging environment. This interaction also entails the question of safety issues for both human and robot during interaction.

- Sufficient knowledge of the context of use: this is for the design of an effective interaction scheme to provide appropriate HRI applications that suit the social/ethnical standards of the user. This also includes the robot's abilities to automatically learn about themselves and their world to well adapt to changing situations.

Experimentations with intelligent robots have repeatedly demonstrated the importance of emotional rewards in robotic applications. Since 2003, several experiments with Paro [16], a seal-like robot, held in Japan, in United States of America, in France, etc., have shown that people (elderly, children) experienced 
some kind of comfortable feeling while interacting and living with robot Paro. In a survey of Forlizzi et al. (2004) [20], emotional rewards were mentioned many times when people talked about the capacity of assistive materials to permanently keep elderly in connection with others (their relatives, their friends, their neighbours, etc.).

Being encouraged from works of psychologists $[1-3,5]$ on the importance of emotional reactions in the adaptation abilities of human, research in artificial intelligence has begun studying the emotional intelligence of human and trying to implement it in robots to render more natural behaviour and so to gain success in interaction with human.

\subsection{Researches in Modelling Emotions For Computerized Applications}

Advances in robotics and artificial intelligence have strongly changed the way people experience computerized applications. With the discovery of emotional rewards for human during interaction with his robotic equipments, research on modelling emotions has come to life. This research direction requires to be based on studies on emotion theories in psychology. Among several theories, some are often used as basis by almost all works. These theories are the theory of Ortony et al [8] on event appraisal, the theory of Lazarus [7] about appraisal and coping, and the theory of Scherer $[3,6]$ about emotional processes.

In the aim of modelling and implementing emotions into computerized application, several approaches have been explored. El-Nasr et al.[11] explored the use of fuzzy logic to calculate emotional intensities. This model was implemented in a simulation named PETEEI - a Pet with Evolving Emotional Intelligence. Embodied Conversational Agents, such as ParleE (by Bui et al, 2002) [12], Greta (Pelachaud, 2003) [17], GALAAD (by C. Adam, 2005) [19], have been developed, taking into account the appraisal process of emotions and even personality traits. There is also a framework for training applications called Mission Rehearsal Exercise, developed by Gratch and Marcella in 2004 [18], that provides virtual scenarios for health intervention, marketing and entertainment. The emotional aspect of this proposition is the adaptation of event appraisal and coping (two important aspects of emotions, according to Lazarus [7]). Now come the applications in the domain of HRI. In 2002, C. Breazeal at MIT lab attempted to establish an interaction between a robot and a human inspired by the relation between a baby and his/her parents [14]. Emotions experimented are anger, distaste, fear, sadness, and happinesss. Recently, MIT lab has also announced their advance in modelling emotions into another robot named Nexi - a Mobile Dexterous Social Robot with more human-centric communication and interaction abilities [15]. In 2008, we proposed a model of emotions for robotic applications, called GRACE $[21,22]$. GRACE is based on the psychological theories that we mentioned previously. This model is considered generic as it can instantiate an-

terior models of emotions in the domain of computer science by using some of its components. A comparison of these models of emotions can be found in [21]. 


\section{Proposition for the Study on Emotional Rewards of HRI Using Music}

The universal appeal of music mostly comes from the emotional reward that music offers to its listeners. According to Zentner in [4], emotional impacts of music explain its prominent role in people's everyday lives. In [2], music is ascertained to have direct effects to treatment of emotion disorders. Now imagine that a robot can detect the emotional state of its partner when he listens to the music (or he plays the music) and then react in an appropriate manner, can this robot enhance emotional rewards for its partner during/after this interaction? This is the question we try to answer in our study.

To this end, beside the study of emotional impacts of music to human, we need a model of emotions to implement the robot's behaviour so as to enable an emotionally rich interaction with humans. With GRACE, we already described and modelled the emotional process for robots. However, the use of music during human-robot interaction requires some specific adaptations. In this section we study these requirements and propose modifications to suit the case of using music.

\subsection{Important Elements in Studying HRI Using Music}

Given that the objective of our work is to study the mental rewards during human-robot interaction using music, the interaction scenario will be between a musician and a robot with music-related abilities. Thus, the desired robot should be equipped with a cognitive process that helps it to perceive its partner's emotions via his music (and maybe his visible behaviour) and then to react in an appropriate way. Important information used for the robot's intelligent behaviour in this study should be:

- The emotional interpretation of the music played by a musician: this represents the interpretation done by the musician. The robot has to know how its partner feels via his music. As claimed in $[4,5]$, it is feasible to construct a learning module that takes a piece of music as input and reproduces the emotions probably experienced by a specific individual.

- The robot's mental state (specifically personality trait of robot: introversive, extroversive, aggressive, curious, etc.). This mental state has an important impact on the selection of action alternatives. So, with the same emotional state of musician (interpreted by the module of interpretation), the robot can change its preferred tendencies of action based on its own mental state. For example, if the detected emotion of the musician is sadness, then the robot, if it is extroversive, can have action tendencies to show empathy to its partner; but if the robot is aggressive, it could have tendency to disturb the musician (like making noises, performing exciting movements, etc.).

- The robot's current goals (excite its partner, get rid of him, disturbing ongoing situation, etc.). This current goal gives hints to select preferred tendencies. When the robot wants to excite its partner, it should prefer actions that decrease the negative emotions of its partner and increase positive impact 
(such as performing funny dances, showing empathy by dancing coherently with the music played by its partner).

- Ability to guess future actions of its partner (the musician). This ability allows the robot to predict upcoming events to better adapt its behaviour. This could be done by deploying a prediction module based on a predefined scenario or applying pattern recognition on the set of events over time.

To take into account important elements presented above, the model GRACE, which remains abstract for general use case, will be adjusted and concretized into an adaptation called MACE-GRACE (Music-adapted Architecture to Create Emotions - GRACE).

\subsection{MACE-GRACE - Adaptation of GRACE in the Context of HRI Using Music}

Being constructed by merging psychological theories on emotions and computational models of emotions, GRACE aims at simulating an entire emotional process of human. The emotional process of GRACE is described in Figure 1:

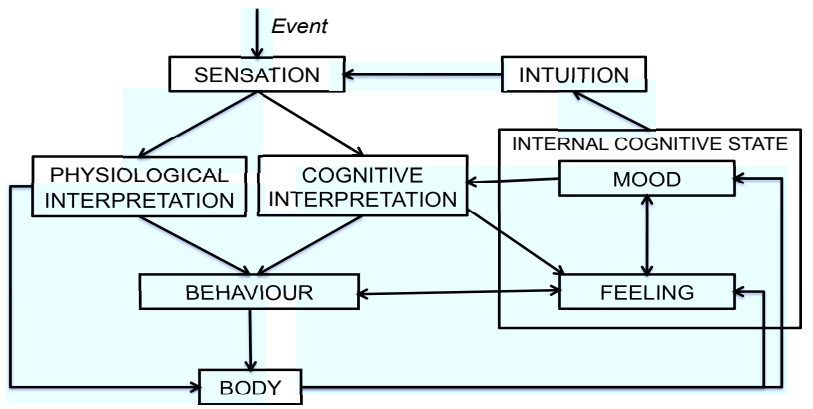

Fig. 1. Model GRACE - Generic Architecture to Create Emotions

For our study on emotional rewards in HRI application, some details must be precisely pointed out:

- Sensation: this GRACE module does the collection of information necessary for emotional processing. In our case, information collected will be musical stimuli (let's call them 'musical events') and environment information required for robot safety verification.

- Physiological interpretation: this GRACE module simulates the physiological symptoms of the emotional process (like temperature sensations, respiratory and cardiovascular accelerations and decelerations, trembling and muscle spasms) to show reflex reactions. In our case, only the safety of the human and the robot will be verified in this module to ensure a good performance of the robot; other physiological symptoms will not be taken into account.

- Cognitive interpretation: in GRACE, this module is in charge of analyzing the emotion-eliciting events to have a global view of current situation (more specifically, the emotion-related aspects of the current situation). Result of this analysis is used to decide action tendencies to respond to events. In 
our case, what we want in output of this module are the current emotions expressed in the musical events played by the robot's partner. We tend to use a learning module here to analyze the musician's emotions via his music. The result of this module can help the robot to choose appropriate actions to respond to the musical events.

- Mood: this component contains the robot's current mental state, its current stand faced to current situation, its current goals/needs in term of assigned tasks and even its habits(e.g. its preferred action tendencies, preferred situation/events). To focus on the goal of our study (mental rewards for humans during the interaction with a robot having music-related abilities), we will code in this module only the robot's goal/need, and a simplified mental state.

- Feeling: this module plays the role of memorization of the running process. It captures the reaction of all components to events and is used as a database for adaptation and memory recall abilities. In our study, this part would store the musician's attitude (history/personality of musician). This module can help the robot to decide its preferred strategy of action in regard to the attitude of musician.

- Intuition: this module of GRACE is supposed to do the anticipation of future events, to generate imaginary events (i.e. internal events). We propose in our study to relate it to some memory recalls or anticipations of future reaction of the musician so that the robot can react rapidly to new events.

- Behaviour: in GRACE, this module is for the selection of an action to respond to an event. This selection takes into account information of both the external situation and the robot's internal state. In our case study, this would be the selection/regulation of robot's movements (accelerate or decelerate the movement of the hands, turn the body around, make some noise, etc.) according to the musician's emotion expressed in his music.

- Body: this module executes the robot's motor expression in response to input events. Generally, this includes reflex reactions from Physiological Interpretation and reactions selected by Behaviour module. In our study, this part will execute the robot's music-related reactions to show that the robot understands the emotional expression in the playing music and shows its response in relation with the current situation and its own personality.

To take into account these issues, we propose MACE-GRACE, an adaptation of GRACE for HRI using music, described in Figure 2. The analysis process of robot's intelligence when it captures musical events will be as follows:

The physiological interpretation is in charge of verifying the physical impact of events to robot to ensure the robot's safety based on its position, its battery level, etc. The simulation of human-like physiological stimuli (such as body temperature, heart beat, respiration, or even reflex reactions) of the robot is not taken into account in this study. The robot's cognitive interpretation will measure its partner's emotions based on the music that he plays. This measured value will be sent to the action selection to determine an appropriate response to the current emotional state (measured by the cognitive interpretation). This phase of selection is also influenced by the robot's current goal/need (calm down 


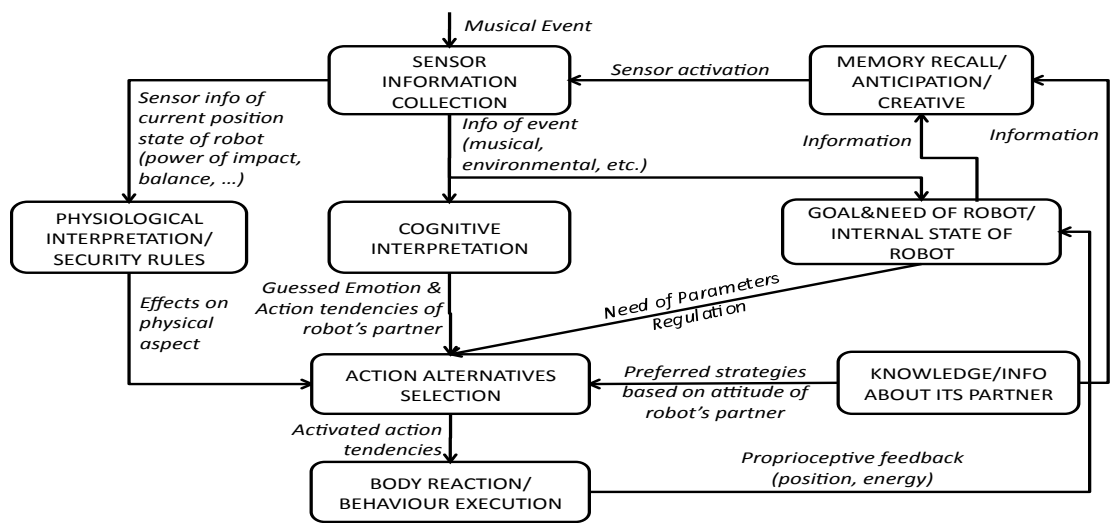

Fig. 2. Adaptation of GRACE in the context of HRI using music

the musician or excite the current ambiance, amplify the current state of the musician) and with regard to the musician's attitude. The execution of the selected action will be stored in the robot's database along with the behaviour of the musician at that moment. This memory allows the robot to make predictions of the future behaviour/music of its partner so that it could have an adaptive behaviour in the whole conversation.

With this adaptation, we tend to integrate in our robot an artificial emotional intelligence to interact with a musician. This emotional intelligence can help the robot not only perceive the emotional state of its partner but also react to diverse situations in an adaptive manner in term of emotion-based strategy.

\section{Conclusion}

Throughout the paper we have presented major characteristics of robotic applications and then mentioned the role of emotional rewards during human-robot interaction. We also proposed an evolution of our model of emotions previously proposed for computerized applications. This evolution is in the aim of studying the emotional rewards in human-robot interaction using music. We consider the study as an attempt to implement emotional intelligence into a concrete computerized application, more specifically, an assistive/entertaining robot. This could have interesting applications in such fields as music-based therapy, emotionbased movements, preferences of robot abilities, etc.

The realization of this adaptation is a long way to go. It consists in implementing the model, doing the experimentation with the robot and then analyzing the results. Furthermore, the implementation of the model could also concern works in database construction (including choices in music style, user preferences, music descriptors, definition of musical event, etc.), system specification, programming tasks, etc. Yet, we demonstrated in this study that GRACE, our generic model of emotions, was versatile enough to be easily adapted to the specific needs of emotional interaction using music.

\section{References}

1. C. Darwin: The expression of the emotion in man and animals. London: Murray, (1872) (Reprinted Chicago: University of Chicago Press, 1965). 
2. S. Gebhardt, R. von Georgi: Music, mental disorder and emotional reception behavior. Music Therapy Today. Vol.VIII (3) (2007)

3. K. R. Scherer: Emotion, the psychological structure of. Smelser, N. J., Baltes, P. B. (Eds.) International Encyclopedia of the Social and behavioral Sciences. Oxford: Pergamon (2001)

4. M. Zentner, D. Grandjean,K. R. Scherer: Emotions Evoked by the Sound of Music: Differentiation, Classification, and Measurement. Emotion, 8(4), 494-521, (2008)

5. K. R. Scherer: Which emotions can be induced by music? What are the underlying mechanisms? And how can we measure them? Journal of New Music Research, $33(3), 239-251,(2004)$

6. K. R. Scherer: On the nature and function of emotion: A component process approach. In K. R. Scherer \& P. Ekman (Eds.), Approaches to emotion (pp. 293-317). Hillsdale, NJ: Erlbaum. (1984)

7. R. S. Lazarus: Emotion and Adaptation. Oxford University Press, (1991)

8. A. Ortony, GL. Clore, L.Collins: The cognitive structure of emotions. Cambridge University Press, Cambridge (1988)

9. Myers, I. Briggs: Gifts Differing: Understanding Personality Type. Davies-Black Publishing; Reprint edition (May 1, 1995). ISBN 0-89106-074-X. (1980)

10. Special Issue of Human-Computer Interaction. Volume 19, Numbers $1 \& 2$, (2004).

11. M. El-Nasr, J. Yen, T. Ioerger: FLAME - A Fuzzy Logic Adaptive Model of Emotions. Journal of Automous Agents and Multi-agent Systems, vol 3, p. 219-257. (2000)

12. T. Bui, D. Heylen, M. Poel, A. Nijholt: ParleE: An Adaptive Plan Based Event Appraisal Model of Emotions. Proceeding of KI 2002: Advances in Artificial Intelligence, 25th Annual German Conference on AI, p. 129-143. (2002)

13. D. Rousseau: Personality in Computer Characters. Proceedings of the 1996 AAAI Workshop on Entertainment and AI / A-Life, AAAI Press, Portland, Oregon,pp. 3843. (1996)

14. C. Breazeal: Emotion and sociable humanoid robots. E. Hudlika (ed), International Journal of Human-Computer Studies, 59, pp.119-155. (2003)

15. Project MDS: Personal Robots Group, MIT Media Lab http://robotic.media.mit.edu/projects/robots/mds/overview/overview.html

16. Official site for Mental Commitment Robot (PARO) http://paro.jp/english/

17. F. de Rosis, C. Pelachaud, I. Poggi, V. Carofiglio, N. De Carolis: From Gretas Mind to her Face: modelling the Dynamics of Affective States in a Conversational Embodied Agent. The International Journal of Human-Computer Studies, vol 59 $(1-2)$. (2003)

18. J. Gratch, S. Marsella: The Architectural Role of Emotion in Cognitive Systems, in Integrated Models of Cognitive Systems. Wayne Gray (ed.), Oxford University Press. (2008)

19. C. Adam: Emotions: from psychological theories to logical formalization and implementation in a BDI agent. PhD Thesis. (2007)

20. J. Forlizzi,C. DiSalvo,F. Gemperle: Assistive robotics and an ecology of elders living independently in their homes. Hum.-Comput. Interact. 19, 1 (Jun. 2004)

21. T. Dang, S. Letellier-Zarshenas, D. Duhaut: Comparison of recent architectures of emotions. 10th International Conference on Control, Automation, Robotics and Vision ICARCV 2008, Vietnam. (2008)

22. T. Dang, D. Duhaut: Experimentation with GRACE, the Generic Model of Emotions For Computational Applications. 2nd Mediterranean Conference on Intelligent Systems and Automation, Tunisia. (Best paper award) (2009) 